\title{
La participation citoyenne en santé mentale: une expérience locale au Québec
}

Jean Gagné - QUÉBEC

Organisateur communautaire au Centre de santé et de services sociaux Jeanne-Mance

\section{RÉSUMÉ}

Cet article présente une expérience de participation citoyenne impliquant des personnes utilisatrices de services de santé mentale dans la localité Jeanne-Mance de Montréal. Il fait tout d'abord état de l'évolution du concept de participation au Québec, depuis le début des années 1960, en reprenant celui d'imaginaire instituant et institué. Ensuite, il présente une expérience de participation citoyenne entendu comme celle des clients des services publics à leur planification et à leur évaluation. Celle-ci a comporté plusieurs activités de recrutement et de promotion publique comme la rédaction d'une politique d'établissement pour la reconnaissance de la participation citoyenne en santé mentale. Cette participation propose un principe de réciprocité malgré une tendance du ministère de la Santé et des Services sociaux du Québec de planifier les soins de manière unilatérale ou top down.

\section{ABSTRACT}

This article presents a citizen participation experience involving mental health service users in the Jeanne-Mance neighbourhood in Montreal. First, it describes how the concept of participation has evolved in Quebec since the early 1960s, reviewing the concept of the imaginary involving the instituting and the instituted. It then presents a citizen participation experience understood as the participation of users in the planning and evaluation of public services. This experience involved several recruitment and public promotion activities such as the writing on an institutional policy of recognizing citizen participation in mental health. Such participation puts forward a principle of reciprocity despite a tendency on the part of the ministère de la Santé et des Services sociaux du Québec (Québec ministry of health and social services) to adopt a unilateral or top down approach to planning healthcare. 


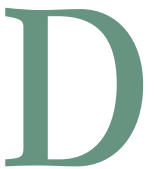

epuis quinze ans, la participation citoyenne ou encore la «nouvelle participation civique» (Santé Canada, 2003) est devenue une véritable mode dans l'univers de la gestion publique. Elle s'impose comme une règle de bonne pratique dans des domaines variés comme le développement international ou la santé publique, et cela tant pour la planification, l'organisation et l'évaluation des services que pour la conduite de recherches sociales et cliniques (Carr, 2004; Santé Canada, 2003). Une revue de la littérature sur son application dans les systèmes de santé, publiée entre 1996 et 2000, concluait qu'il y avait encore assez peu de preuves confirmant que la participation citoyenne avait un impact significatif sur la qualité des systèmes de santé (Crawford et al., 2002).

$\mathrm{Au}$ Canada, un examen des pratiques en santé mentale conclut pourtant que: «Tous les programmes et stratégies fondés sur les meilleures pratiques mettent l'accent sur la participation des usagers à leur planification, à leur gestion et à leur évaluation » (Institut psychiatrique Clarke, 1997). Pour discuter des grandeurs et misères de l'application de ce concept en situation réelle, le présent article s'appuie sur une expérience d'intégration de personnes utilisatrices d'une instance consultative de planification des services de santé mentale dans un réseau local de services du centre-ville de Montréal.

\section{Historique de la participation citoyenne en santé mentale au Québec}

Au Québec, le Plan d'action en santé mentale 2005-2010 - La force des liens (PASM) (MSSS, 2005) s'est fait le porteur de cette nouvelle participation civique grâce à «la participation des utilisateurs et des proches aux exercices de planification des services de santé mentale». Le PASM précise que les centres de santé de services sociaux (CSSS) sont responsables de «définir les mécanismes qui conviennent pour obtenir cette collaboration». Ce mandat est cohérent avec la création des réseaux locaux de services de santé composés des différents dispensateurs d'activités et de services sociaux ou de santé, les organismes communautaires, les entreprises d'économie sociale et les ressources privées sans oublier les CSSS chargés de leur coordination. Le PASM indique que tous «les partenaires du domaine de la santé mentale sont appelés à favoriser la participation des personnes utilisatrices de services au regard du mandat qu'ils assument, tout en offrant des conditions d'exercice adaptées aux capacités de ces dernières ».

Cette nouvelle reconnaissance de la valeur des savoirs profanes serait attribuable au relatif échec des «experts» à résoudre les problèmes sociaux et de santé ainsi qu'au besoin des administrations publiques de renflouer leur légitimité largement remise en question par le contexte de mondialisation des communications, de l'économie et des institutions réglementaires. Dans ce nouveau cadre, les décisions se prennent par des nonélus et «ailleurs» que dans les parlements. Les citoyens réagissent par un certain cynisme envers la politique et les autorités qu'elle institue. Dès lors, on parle d'un déficit démocratique que la participation locale et sectorisée pourrait combler en tant qu'elle se présente comme un lieu concret où le citoyen peut exercer une emprise sur les décisions qui le concerne (Barnes et al., 2007). Comptetenu de l'époque où apparaît la nouvelle participation civique, nous pourrions être tentés de l'associer essentiellement à la rationalité néo-libérale. Elle ne jouerait alors qu'un rôle d'adjuvant, pseudo démocratique, à une planification sociale étatique, voire même mondiale pour ce qui est des programmes issus d'organismes internationaux de développement.

Mais s'il y a une «nouvelle» participation civique, il y en a forcément une ancienne qui pourrait nous rappeler les objectifs ayant prévalu à son évolution et aux sources de tension qui traversent aujourd'hui cette nouvelle participation, perçue à la fois comme émancipatrice et aliénante. Il y aurait, d'une part, de nouvelles demandes sociales qui cherchent à s'instituer comme normes et, d'autre part, une institution qui, pour se maintenir, tendrait à encapsuler cette nouveauté, à se l'approprier et à la reformuler dans les termes de sa représentation de l'ordre et de la signification du monde (Castoriadis, 1975).

L'idéologie de la participation «s'est épanouie au printemps de la Révolution tranquille», c'est-à-dire en pleine élaboration de l'État providence au Québec qui fut, tout 
comme la participation civique d'aujourd'hui, porté par un consensus paradoxal entre des planificateurs experts recrutés par l'État et des activistes promoteurs d'une démocratie directe. Le mot d'ordre de participation a inspiré la création des comités de citoyens revendicateurs des retombées de ladite révolution. C'est aussi sous son égide que s'est institutionnalisée la participation de groupes issus de la société civile, à l'implantation de plans de réaménagements ruraux et urbains ainsi qu'à celles de réformes en éducation ou en santé. Ce mouvement d'appropriation citoyenne a donné lieu à la formation d'une panoplie d'organismes communautaires.

Il s'est depuis maintenu et développé en tant que dispensateur de services alternatifs ou complémentaires au réseau public. Cette évolution s'est faite en trois temps comme dans le reste du Canada, aux États-Unis et même en Israël. Il s'agirait bien d'un processus d'institutionnalisation qui, dans les années 1960-1970, a pris la forme d'un syndicalisme de cadre de vie pour ensuite se développer en tant que secteur alternatif aux services publics pour enfin, depuis le milieu des années 1980, s'intégrer à une planification étatique plus ou moins négociée.

Dans le champ de la santé, en particulier, la publication des rapports du Comité Brunet sur les CLSC (Gouvernement du Québec, 1987a) et du Comité Harnois (Gouvernement du Québec, 1987b) sur la politique de santé mentale en 1987, puis celui de la Commission Rochon en 1988 (Gouvernement du Québec, 1988) s'accordent sur la nécessité d'une reconnaissance par l'État des organismes communautaires. Cette même recommandation a été reprise dans la Politique de santé mentale du Québec de 1989, adoptée en 1991 par la loi 120 (Art, 335, LRQ, chap. S-4.2). La nouvelle définition des rapports entre l'État et les organismes communautaires a été confirmée par la Politique gouvernementale de reconnaissance de l'action communautaire adoptée en 2001. Enfin, depuis 2003, la loi 25 sur le développement des réseaux locaux de services de santé et de services sociaux confie aux instances locales ou centres des services sociaux et de santé (CSSS), la responsabilité de l'élaboration d'ententes de services avec les organismes communautaires qui sont inclus de facto dans les réseaux locaux de services que chacun des CSSS doit coordonner.

Lidée de participation de la communauté à la planification et à l'organisation des services, comme on le voit, perd de son sens politique pour tendre de plus en plus à être une affaire administrative où la communauté n'est conçue que comme un ensemble de corporations privées, à but lucratif ou non, qui produisent des services sociaux et de santé dans une aire géographique donnée.

Avec le PASM, l'idée de la participation réapparaît centrée sur l'individu ou sur la personne utilisatrice de services. Le danger est de réduire le sujet politique au sujet qui consomme et, en corollaire, le bien public aux services publics. Il existe aussi un risque de fragmentation de la citoyenneté en autant de «clientèles» sous le prétexte d'une égalité formelle de leurs intérêts variés et surtout de leur capacité à les défendre (Barnes et al., 2007). Par contre, cette nouvelle participation pourrait aussi être une autre voie pour faire surgir la «voix oubliée» des personnes que l'on prétend soigner et remettre avec elles en question nos objectifs, transformer nos pratiques et réévaluer nos critères de qualité, bref, pour bouleverser notre imaginaire institutionnel.

\section{Un exemple d'expérience locale en santé mentale}

Lexpérience locale de soutien à la participation citoyenne en santé mentale présentée ici existe depuis quatre ans. Elle s'inscrit dans le cadre de l'implantation locale du PASM et dans le projet d'intégration des services du MSSS qui prévoit que: «La gestion [du système de santé et de services sociaux] sera orientée en fonction de cibles précises, résultant des priorités ministérielles et visant à améliorer la santé et le bien-être de la population de même que l'efficacité des services» (MSSS, 2004). Cette formulation laisse peu de place aux initiatives et à la créativité. Toutefois, la responsabilité dévolue aux CSSS de planifier les services en concertation avec la population lui confère un levier qu'elle peut utiliser pour instituer localement des innovations.

C'est dans ce cadre qu'est né, en 2008, le groupe d'action et de réflexion sur la participation citoyenne en santé mentale dans 
Jeanne-Mance, le GARE, pour assister le Comité conseil sur le plan de l'organisation clinique du programme santé mentale adulte du CSSS Jeanne-Mance. Ce groupe fut créé suite à la participation en 2006 d'un représentant du collectif montréalais de défense des droits en santé mentale ActionAutonomie sollicité par l'établissement pour siéger au Comité conseil. Il vise à éviter l'isolement et la marginalisation dont sont victimes les personnes aux prises avec un trouble mental en se constituant comme un groupe d'appartenance. En réponse aux demandes de ce groupe, le CSSS a accepté de doubler le nombre de représentants et a désigné une personne substitut en cas d'absence de l'un ou l'autre des déléguées officiels. De plus, le CSSS a mandaté une personne pour faire le lien avec le collectif de défense des droits et co-animer des «rencontres d'info-consultation» (Labesse, 2009) avec les différents organismes œuvrant en santé mentale ou des populations susceptibles d'utiliser des services de santé mentale. Ces rencontres ont alimenté la contribution des délégués utilisateurs du Comité conseil en santé mentale du CSSS. De plus, ces visites ont permis le recrutement d'autres personnes utilisatrices pour former le GARE.

Cette association est devenue un groupe de pairs pour soutenir les personnes utilisatrices déléguées au Comité conseil et un lieu de propositions et de revendications. Le GARE a ainsi piloté l'organisation et l'animation d'un atelier de formation sur le logement et la santé mentale et a présenté une communication aux journées biannuelles en santé mentale organisées par le MSSS en mai 2009. Dès lors, un certain nombre des conditions de participation (Perkins, Goddard, 2005; Carr, 2004; Binet et al. 2004) se mettaient en place pour soutenir la participation des usagers dans la planification des services de santé mentale: une participation en tandem, un support technique et professionnel externe, des moyens concrets pour consulter et délibérer entre pairs.

Afin de donner un caractère durable à cette expérience, les personnes utilisatrices déléguées au Comité conseil du CSSS ont proposé de créer un comité de travail multipartite pour rédiger une politique de participation des personnes utilisatrices pour la planification et la dispensation des services en santé mentale. Après une année de pourparler, cette proposition a été approuvée par le Comité conseil et entérinée par la direction du programme. Un texte a été produit par un comité de travail formé majoritairement de personnes utilisatrices en collaboration avec des représentants du CSSS, des organismes communautaires autonomes et des services hospitaliers de la localité. Il demande à l'institution de s'engager à maintenir ses pratiques de participation par delà la durée des planifications locales ou nationales pour en faire un élément central de son mode habituel de gestion. Il propose que l'établissement élabore, lors de chaque collaboration avec des citoyens participants, une entente qui en précise les objectifs et modalités de travail ainsi que le traitement et le statut qui seront accordés aux productions attendues. Il recommande aussi que soit accordé aux participants un remboursement de leurs frais liés directement à leur participation ainsi qu'un montant forfaitaire en reconnaissance de leur contribution citoyenne et de leur expertise. Enfin, il souhaite qu'un suivi participatif et évaluatif de l'expérience soit réalisé. Ce texte est en attente d'une approbation institutionnelle.

\section{Conclusion}

Malgré qu'il réponde à une orientation du MSSS, cette expérience de participation réalisée à Jeanne-Mance demeure assez marginale. Le collectif Action-Autonomie de Montréal révélait, en 2008, que quatre des douze CSSS de la région montréalaise entretenaient des mécanismes de consultation auprès de la population utilisatrices pour l'implantation de leur plan local d'organisation clinique en santé mentale. Cette «résistance» institutionnelle est attribuable à la «dissonance culturelle» que provoque la participation en modifiant les rapports usuels entre protagonistes: usagers, praticiens et gestionnaires. D'abord, ce nouveau rapport se situe dans un registre politique et collectif qui fait contraste avec le colloque singulier de la clinique. Ensuite, il remet en question la hiérarchie des pouvoirs et des savoirs entre l'intervenant et la personne utilisatrice pour la remplacer par un principe d'égalité et de coopération. Il faut enfin reconnaître qu'il s'agit d'une figure imposée, mais de manière si peu explicite qu'il 
est facile de la contourner en se limitant à des mesures minimales, formelles et peu investies.

Mais par delà les investissements que l'établissement pourra faire pour mobiliser la population, il faudra encore que celleci y trouve son compte. Or, les CSSS sont imputables de l'atteinte des objectifs fixés par le MSSS dans le cadre des ententes de gestion et cela conditionne leur financement. Dans ce cadre de gestion "par en-haut", la participation locale peut se réduire à de l'information, voire au «marketing» de la réforme. Le projet de politique de participation dans Jeanne-Mance propose à l'inverse que la participation soit considérée comme une pratique de réciprocité. Il s'agirait non plus seulement d'amener la population vers le réseau public afin que celle-ci s'insère dans son paradigme de gestion clinique et organisationnel, mais plutôt de construire un processus où la population pourrait questionner les priorités et les façons de faire, voire ré-imaginer son institution.

\section{Bibliographie}

Barnes M., Newman J., Sullivan H. (2007). Power, Participation and Political Renewal Cases Studies in Public Participation, Bristol, The Policy Press, 228 p.

Carr S. (2004). Has service user participation made a difference to social care services? London, Social Care Institute for Excellence, $42 \mathrm{p}$.

Castoriadis C. (1975). L'institution imaginaire de la société, Paris, Seuil, 538 p.

Binet L., Clément M., Labelle M.H. et Comité d'orientation de l'APUR (2004) Les utilisateurs de services de santé mentale évaluent leurs expériences de participation... Un premier bilan, Québec, APUR/GRIOSE, 80 p.

Crawford M.J., Rutter D., Manley C., Weaver T., Bhui K., Fulop N., Tyrer P. (2002). "Systematic Review of Involving Patients in the Planning and Development of Health Care", British Medecine Journal, 325: 1263.

Gouvernement du Québec (1987b). Pour un partenariat élargi: projet de politique de santé mentale pour le Québec, Québec, Comité de la politique de santé mentale, MSSS, 185 p.

Gouvernement du Québec (1988). Rapport de la commission d'enquête sur les services de santé et les services sociaux, Québec, Les Publications du Québec, 803 p.

Gouvernement du Québec (1987a). Rapport du comité de réflexion et d'analyse des services dispensés par les CLSC, Québec, MSSS, 185 p.

Institut psychiatrique Clarke - Unité de recherche sur les systèmes de santé (1997). Examen des meilleures pratiques de la réforme des soins de la santé mentale - Analyse situationnelle, Ottawa, Travaux publics et Services gouvernementaux Canada, $130 \mathrm{p}$.

Labesse M.E. (2009). «Prenez GARE aux citoyens de Jeanne-Mance», Développement social, $10(1): 29$.

Ministère de la Santé et des Services sociaux (2005). Plan d'action en santé mentale 2005-2010, La force des liens, Québec, Gouvernement du Québec, 96 p.

Ministère de la Santé et des Services sociaux (2004). L'intégration des services de santé et de services sociaux. Le projet organisationnel et clinique et les balises associées à la mise en auvre des réseaux locaux de services de santé et de services sociaux, Québec, Gouvernement du Québec, 26 p.

Perkins R.E., Goddard K.L. (2005). "Involving Service Users and their Relatives”, in James A., Worrall A., Kendall T., (éds), Clinical Governance in Mental Health and Learning Disability Services - A Practical Guide, London, Royal College of Psychiatrists, 131-148.

Santé Canada (2003). Exploration du lien entre la participation du public-L'engagement des citoyens et les soins de santé de qualité - Examen et analyse de la documentation existante, Ottawa, Santé Canada, 69 p. 